\title{
The Application of Variation Translation in Song Dubbing
}

\author{
Yun Xiang \\ School of Foreign Languages, Hubei University for Nationalities, Enshi, Hubei, China \\ 52642950@qq.com
}

Keywords: Variation Translation, Song Dubbing, Application

\begin{abstract}
Song dubbing, a special branch of literary translation, refers to the translation of songs that can be sung. Variation translation is the translation activity of the translator adopting alternative and flexible means to meet the specific needs of certain readers under certain conditions. Song dubbing is the art of translation, involving literature, music and translation. Therefore, the text translation of songs will be subject to the customs and cultures, the trend of the melody, the rhythm of music and many other factors. And the translator has to take the corresponding flexible and variable skills to meet the standard of song dubbing: "to sing", "to hear" and "to empathize".
\end{abstract}

\section{变译策略在歌曲译配中的实践运用}

向云

湖北民族学院外国语学院, 恩施, 湖北, 中国

52642950@qq.com

关键词: 变译, 歌曲译配, 实践运用

摘要：歌曲译配是指能够配曲而唱的歌曲翻译，属于文艺翻译中的特殊分支。而变译 “是译者 据特定条件下特定读者的特定需求，采用变通手段摄取原作有关内容的翻译活动”。歌曲译配是 戴着 “紧䈐咒” 的翻译艺术, 是集文学、音乐与翻译于一体转换的艺术。因此, 歌曲文本在转换 中会受制于民俗文化、音乐中的节奏和旋律走向等等多种因素，在歌曲译配中，译者不得不采取 相应的变通策略以满足译配后的歌曲 “可唱”、“可听”、“可感”的要求。

\section{1. 歌曲译配}

歌曲译配研究是近二十余年才受国内学术界关注的研究内容（始于 1993 年）。近五年来, 歌 曲译配研究才得到较大发展, 文献多以 “歌曲翻译” 出现, 占 326 篇, 以 “歌词翻译” 为篇名的 占 191 篇, 以 “歌曲译配” 与 “歌曲配译” 为篇名的仅占 48 篇, 其中硕士论文 46 篇, 博士论文 1 篇。相关专著仅有三部: 孙慧双的《歌剧翻译与研究》 [1]、薛范的《歌曲翻译探索与实践》[2] 及笔者的《英汉歌曲译配: 理论与实践》 [3]。薛范先生[2]将 “歌曲翻译” 定义为: “把原词用诗 的形式翻译出来，并且与原曲的节奏、旋律等相吻合，即配上曲子可以歌唱”。在薛先生的书 里, 歌曲翻译等同于歌曲译配, 有别于未曾配曲、无法歌唱的歌词翻译。他自己也承认这样的分 类含混不清, 是不科学的。胡风华老师[4]认为 “歌曲翻译” 是一种俗用、通用, 多数指 “歌曲译 配” , 有时指 “歌词翻译” ; 但从音乐学和翻译学角度看, 只有 “歌曲译配” 才能既包括译词又 包括配曲, 才是地道的专称专用。笔者[3]把歌曲译配定义为 “能够配曲而唱的歌曲翻译, 属于文 
艺翻译中的特殊分支”, 并厘清了歌曲翻译与歌曲译配的关系, 认为歌曲翻译是歌曲译配的上位 词。在国外, 涉足歌曲译配研究的相关 SSCI 论文不足 30 篇, 远少于国内, 但随着视听翻译在西 方越来越受重视, 近些年国外学界共出版歌曲译配相关论文集一部 Translation and Music [5], 著 作 5 部: Song and Significance: Virtues and Vices of Vocal Translation [6]、Sounds in Translation: Intersections of Music, Technology and Society [7]、Music, Text and Translation [8] 、 Translating for Singing: the Theory, Art and Craft of Translating Lyrics [9]、Translating Song: Lyrics and Texts [10]。

\section{2. 变译策略}

变译是 “译者将原语文化信息转换成译语文化信息以满足读者特定需求的思维活动和语言活 动” [11]。变译是相对于全译而论, 是翻译的两个新范畴, 从 “保全原作内容和形式” 的程度来 分, “力求保全” 的为全译, “有所取舍和改造” 的为变译。同样, 黄教授[11]在其《变译理 论》一书中这样阐述到：“变译是译者据特定条件下特定读者的特定需求，采用增、减、编、 述、缩、并、改等变通手段摄取原作有关内容的翻译活动”。从中看出, 它凸显了变译不同于全 译的最大特征就是 “变”，揭示了 “为谁变”，“谁来变”，“对谁变”，“怎样变”，“为何 变” 等等问题的实质。变译是为读者而变, 目标很明确, 动机很清晰, 翻译的一切就是为读者服 务。读者的需求不同，翻译也应不同，随需求变化而变化，因而变译活动也 “千姿百态”。变译 活动由译者来变, 应读者不同的特殊需求采取变通策略。变译活动针对原作而变, 对原作进行变 通或同时进行翻译的活动。

变译是相对于全译提出来的, 二者并非对立, 而是相互并存, 有效补充, 变译中有局部全 译，全译中有微型变译。变译中的一个核心理论就是 “变通”，与 “迎合”、“通融” 相近，是 寻求双方和多方都能接受的妥协方案和折衷办法的行为, 决非随意而为, 其运用之妙是一种高超 的艺术[12]。

\section{3. 歌曲译配的几种变译体式}

歌曲译配包括两个行为，“译” 和 “配”。受制于 “配” 的过程，歌曲的 “译” 就不得不采 取变通, 或增或减, 或编或改等等才能最终达到歌曲的可唱, 可听, 可感 [3]。杨晓静[13]在其博 士论文中根据译歌对原歌变通幅度的大小将歌曲变译方法归纳为: 译写、删译、改译、摘译、形 译、译述和仿作。而“形译”和“译述”没有“译配”的要求, 故不在本文关于歌曲译配讨论的范畴。

（1）译写，即增加词、语或句的变译，是指在歌曲译配中为了满足译词与原歌在音节数和节 奏上相符, 译配者采取增加原歌词中没有的内容和形式。除去少部分汉语虚词可以与其他实词定 为一个音节外，汉语的音节数是一个词可以定为一个音节。而英语音节数的划分是以元音为单 位，一个元音一个音节，所以英语单词可分为单音节词，双音节词和多音节词。译写方法的原则 是既增形又增意，是 “无中生有” 的增添，在译语歌词中增添原歌曲中没有的内容，以满足音节 数的一致，但无中生有并不等于可以任意而为、胡乱增添，是在符合译语表达方式且与整体音乐 风格一致的前提下进行增加变通。因此，在表达内容相同的情况下，汉语音节数少于对应的英语 音节数。为了不影响歌曲节奏, 在英语歌曲译配为汉语歌曲时, 只能增添一些词、语或句等语言 单位，虽与原歌曲意义无关但符合汉语表达且与整体音乐风格一致。

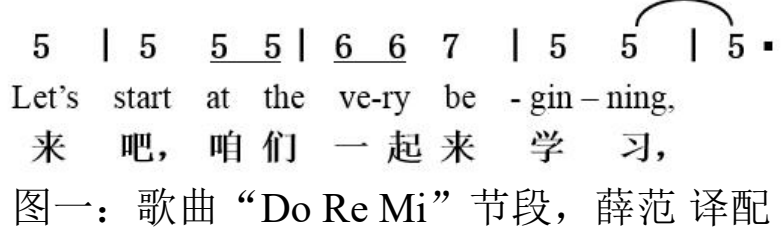


本例中, 英语原意为: “让我们从头开始” ，但开始什么呢？语义上不通，增加了名词 “学 习”，同时把 “让”删减掉，增加原来没有的动词 “来吧”，与其节奏、整体音乐风格相符。英 语音节数为九个音节, 为了汉语音节数也为九个, 于是就凝练成了 “来吧, 咱们一起来学习”。 合上旋律，增词毫无违和感，同样符合原歌曲轻快、活波的旋律风格。

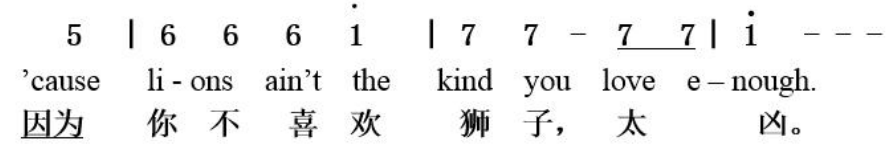

图二：歌曲 “(Let Me Be You) Teddy Bear” 节段 薛范 译配)

例中的汉语译文把原句分成了两个乐节, 第一个乐节节奏型为 $\mathrm{X}|\mathrm{XXXX}| \mathrm{XXX}$ 。第二个乐 节的节奏型为 $\mathrm{X} \mid \mathrm{XXX}$ 。整个英文句子““cause lions ain”t the kind you love enough”压缩成汉语“因 为你不喜欢狮子”，共 8 个音节，本来刚好可以和节奏吻合，但这样 “因为” 的 “为” 字就放在 第一个重拍上了, 出现 “断句”, 译者只好处理成“因为”共一个音节。第二个乐节, 薛先生加上 原文没有的内容 “太凶”，与上句语义融会贯通，“无中生有”，但却增添得“有理有据”。

(2) 删译, 即删减词、语或句的变译, 在歌曲译配中为了满足译词与原歌在音节数和节奏上 相符, 译配者采取删减原歌词中语言单位的内容和形式。译配歌曲时, 译词音节数超过原文音节 数或译文音节不符合原文节奏和旋律走向时, 译者就要采取删译, 既减形又减意, 删掉多余的词 语、句子且不影响整个主要思想内容的表达。

我的那个哥嫂（噻）已经分了家

My dear elder brother is living on his own

（《六口茶》“Six Sips of Tea” 节段）

在此句歌词中哥嫂并没有翻译成 “elder brother and sister-in-low” 而是删译为 “elder brother” , 并没有影响主要内容的表达。在翻译过程中, 译者删减英语歌词中某些语言内容和形 式，以求译文歌词音节数与原作音节数相符，这样歌曲才具有可唱性。

九月重阳酿黄酒咯

September's good for making wine

醉人的黄酒抿（呀）抿（个儿）甜呢

The wine tastes good, smooth and sweet, elegant and fine

(《幺妹, 叭一口》, 覃军 译配)

这是民歌《幺妹，叭一口》节选片段，由覃军译配。因为是恩施洲土家族民歌，歌词具有浓 郁的民族地方色彩, “抿”、“叭”这两个本土方言在翻译中只能最大限度地接近其主要含义, 故 “抿”翻译为“taste”。第一个分句“九月重阳酿黄酒咯”里囊括三个名词，“九月”、“重阳”和“黄 酒”, 如果都直译出来, 英语音节数就远远超过汉语音节数。而 “重阳”节（Double Ninth Festival）指的是每年农历“九月初九”，具有浓厚的文化深层含义，更是被历代文人墨客所歌 咏, 那句千古绝唱 “每逢佳节倍思亲” , 就指的是这个时候, 代表“重逢、团聚、感恩、思念”。 可这么大的信息量不可能在歌曲译配中受制于音节数而翻译出来，另外即使翻译出来，也不符合 译语民族文化表达习惯，在国外，没有对等物，也就没有对应词。综合考虑之下，译者只能“忍 痛割爱”删译“重阳”一词，用前面“九月”大致交代出时间背景。同样是受限于音节数的影响， “黄酒”就用“酒”（wine）来指代, 因为对于目标语听众, 黄酒就是一种酒, 到底是什么酒已不是 很重要。所以也只能采取删译的变通策略, 整句译为“September's good for making wine”, 八个音 节与汉语音节数一致。这种删译理解为“文化性删译”, 为符合译语民族文化所接受和理解的表达 
方式而变通。第二句采取的变通手法更多, 有删译“醉人的”, 有译写增添 “good, smooth, elegant and fine”。总之, 本例的删译与译写的增添都是把听众需求放在第一位, 以满足听众的习惯表达 和文化接受度为目标。

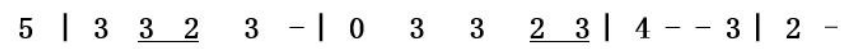

$$
\begin{aligned}
& \text { My day in the hills has come to an end, I know. } \\
& \text { 流连在山上, 眼看暮 色 苍茫。 } \\
& \text { 图三: 歌曲 “The Sound of Music” 节段 薛范 译配 }
\end{aligned}
$$

本例为四四拍，两个乐节，英语原文“My day in the hills has come to an end, I know”可释义为 “流连在山上，但归期已至，我知晓”。汉语音节数大大超过了英语音节数，薛先生便删减掉次 要内容“我知晓”与“归期已至”, 同时译写出“眼看暮色苍茫”。在这删减与增添之间变通开来, 追 求最终译配歌曲能唱且能被听众所领会。

（3）改译，即改写式变译，指在歌曲译配中，为满足译配歌曲“可唱”、“可听”、“可 感” 且译语符合目标语的表达习惯，译配者采取改写原语语言内容和形式的变通策略。这种变译 方法在歌曲翻唱的译配活动中尤为常见。改译的最大极限便是改写得“面目全非”，常常被称之为 重新填词，曲还在，词却全部换掉，也是“旧曲新词”的典型译配活动。改译到极致的成功范例不 胜枚举。歌手们借用外国歌曲翻唱和电视音乐剧中引进国外经典曲目时, 注入了歌曲的“第二次 生命”，同时也可捧红翻唱歌手，是流行音乐创作中比较快捷，省时省力的一种常见手段。当 然, 也有人通过改译变通, 关注社会焦点, 对原作进行恶搞的也不乏其例, 主要是在当前网络媒 体的推动下，这样的音乐恶搞蔓延速度和广度都较大。但一般的歌曲译配还只是局部改译，以求 句子通顺、语意连贯、逻辑清晰、符合译语民族文化习惯表达。

正月里是新年哪咿呦喂

Spring sends away the old year, brings in the new

妹娃我去拜年哪呵喂

I am going for visits on th' road

金哪银儿索 银哪银儿索

I see early birds, do you see th' early birds?

那阳鹊叫啊是 捎着莺鸽啊 捎着莺阿鸽

They are singing that what a pretty girl. Any one knows her?

白:妹娃要过河 是那个来推我吗?

A river lies here, how can I get there?

答:我来推你嘛

I'll ferry you there.

捎公你把舵搬哪 妹娃子我上了船

You hold the rudder so tight, I look up into your eyes

啊喂噎唑 啊喂噎唑 将阿妹推过河呦 呵喂

Ten miles to go, one mile to go. The river kisses the boat in soul.

(《龙船调》翻译节段 覃军 译配)

整首歌，歌词不多，有很多拟声词、衬词、“本土”词汇，所以有人认为这类特色词汇应该在 翻译中保留下来。倘若将其音译直接保留也冊庸置疑, 但这样所剩下的的歌词内容就太简短了, 对译语听众（读者）产生的效果也可想而知，不符合英语表达习惯和英语本族人的认知规律。当 然其实即使译了出来, 在译文中也失去了原文的味道, 所谓有得必有失。故译者尽力在挖掘词句 之情、言外之意。这属于改译的幅度最大的一首歌曲。“咿呦喂”、“呵喂”改译为“brings in the 
new”、“on th’ road”，承接上句补充具体，与上句融会贯通，再加上音节数巧妙的对应也算可圈 可点。同样, “金哪银儿索银哪银儿索，那阳鹊叫啊是捎着荣鸽啊捎着莗阿鸽”已改译的“面目全 非”, 译为 “I see early birds, do you see th' early birds? They are singing that what a pretty girl. Any one knows her?”。在逻辑上承前启后, 为“妹娃要过河是那个来推我吗? ”这句做好内容上的铺 垫。最后两句 “捎公你把舵搬哪, 妹娃子我上了船, 啊喂噎唑, 啊喂噎唑, 将阿妹推过河, 呦呵 喂”译为“You hold the rudder so tight, I look up into your eyes, Ten miles to go, one mile to go. The river kisses the boat in soul”，可见译文与原文也是“相去甚远”，但从整体布局来看，讲述的妹娃 儿的这份“娇羞”与艄公的“野性”，译文将其尽显得淋漓尽致，尤其是这份“挑逗式情谊”用 “river”、“boat”和“kiss”三个词呈现出的画面同样让人耐人回味、经久不绝。

张家溜溜的大哥看上溜溜的她吆

A young man of her neighbor has fallen in love with her

月亮 弯 弯

The moon ... shining

看上溜溜的她吆

Over the town of Kangding

(《康定情歌》翻译节段 覃军 译配)

这个节段中 “张家溜溜的大哥”，没有释义为: “The elder brother of the Zhang's”，而是先删 译了祄词“溜溜的”，再改译为“A young man of her neighbor”。在符合译语民族文化以及语言习惯 表达基础上，更改了原歌曲中某些语言单位的意义，从整体逻辑上、音节数上、节奏上基本都能 与原歌相符，具有可唱性。同样“看上溜溜的她哟”并没有像原作那样重复为“has fallen in love with her”，而是改译成“over the town of Kangding”，承接上句，将上句语义补充完整。改译后语 义明确, 逻辑清晰。这也体现了英语为形合语言这一规律, 注重语法形态、句与句之间的逻辑关 系。

多少春去春来朝朝暮暮

Years after years and days come and days go

来生来世还做你的狐

I'm still right here waiting for you

(《白狐》翻译节段 覃军 译配)

“来生来世还做你的狐”基本上完全改译成“I’m still right here waiting for you”，符合译语文 化及表达。在整个 《白狐》这首歌里, 白狐并没直译为“white fox”, 而歌曲名《白狐》也是改译 为 “The Fox Lover”, 因为整首歌曲唱的是“相爱相离”的主题, 并非是描绘动物之类。改译后, 其整体语义与音乐风格仍基本与原作相吻合。改译的句子也并非凭空乱改，而是与上下语句相关 联。

（4）摘译, 即摘取歌段或声部的变译, 是为了符合译语文化里的演唱习惯和译语歌曲的常见 规范, 译者所采取摘取的变通策略。在摘译中, 遵从“摘主不摘次”原则, 即摘取主要内容或主要 声部。

如《龙船调》的版本很多，又叫《种瓜调》，从正月唱到十二月，唱的是《十二月种瓜》， 后经改编, 以二段歌词和三段歌词常见。译者往往采取摘译的方法, 翻译两段歌词。因为无论有 几段歌词，后面段落的内容上基本只变换了时间和事件。也就是前两句稍有变化，后面的故事内 容和情节都未做调整。几个段落都翻译出来, 意思上显得多余圥长。 
（5）仿作, 即重新填词的变译, 歌曲旋律不变而歌词内容会以原作为依托, 译者将其重新改 写的变译方法。仿作的作品除旋律和曲风与原作相同或相似外，仿作的内容可以保留原作信息一 部分也可以完全不保留, 但要保持整体音乐风格和情感基调与原作基本一致。

Doe, a deer, a female deer //Ray, a drop of golden sun //Me, a name I call myself //Far, a long, long way to run //Sew, a needle pulling thread //La, a note to follow Sew //Tea, a drink with jam and bread...

哆是都来一起唱, 来(ray)是花芯有花香, 咪是猫儿咪咪叫, 发是头发黑又长, 嗦要锁在大门 上，拉起手儿多欢畅，西水渌渌清又凉......

（歌曲“Do Re Mi”节段 程何 译配）

原歌词在语言处理上很巧妙, 为方便儿童记忆, 便借助与音符名相近的英语单词进行遣词造 句。如 “ 1 ” 的发音和英语单词 “doe” 相似, “ 2 ” 和英语单词 “ray” 相近, 以此类推, 每个单 词一一解释, 让歌词顿生趣味且过耳不忘, 孩子们在快乐的歌唱中得到音乐启蒙, 寓教于乐。运 用的英语词汇当然是儿童们熟悉浅显绝不生僻的词汇。那么程何在译配过程中, 也遵循这一原 则，找到每个音符的汉语同音词，同时用浅显易懂的词语将汉语同音词连词成句。如音符 “ 1 ” 和汉语 “哆” 读音相近，音符 “ 2 ”与 “来” 读音相近，音符 “ 3 ”与 “咪” 读音相近。“咪” 造 句为 “猫儿咪咪叫”, 既生动又符合儿童用语, 便于记忆、歌唱。虽从意思的角度看, 原作与译 作已无关联。但却能达到原听众对原歌曲的反应与中国听众对汉语版本的反应相似的效果, 追 “神似”，入 “化境”。可见，仿作的作品与原作整体风格和基调是一致的，内容相差虽大，但 符合译语文化表达习惯，自然、生动，更能让译语听众所接受。

\section{4. 总结}

译写, 删译, 改译, 摘译和仿作是歌曲译配中采取的常见变译方法, 是行之有效且能指导实 践的策略方法。歌曲译配受制于多重因素，在实践中为了满足特定需求和目的，符合 “可唱” “可听” “可感”的歌曲译配原则, 译者不得不采取相应变通策略。

\section{基金项目}

本文系 2018 年湖北民族学院院内青年科研项目 “基于语料库的中国民族歌曲译配研究” (MY2018Q014）成果。

\section{参考文献}

[1] 孙慧双. 歌剧翻译与研究 $[\mathrm{M}]$. 武汉: 湖北教育出版社, 1998.

[2] 薛范.歌曲翻译探索与实践[M].武汉：湖北教育出版社，2002: 31.

[3] 向云. 英汉歌曲译配: 理论与实践 $[M]$. 广州: 世界图书出版广东有限公司, 2017:98.

[4] 胡凤华. “歌曲译配” 与 “歌曲翻译” 辨[J]. 安徽大学学报（哲学社会科学版），2007: 96-100. [5] S.Susam-Sarajeva, "Translation and Music: Changing Perspectives, Frameworks and Significance,"Translator, vol. 14, 2008, pp.187-200.

[6] D. Gorlee. Song and Significance: Virtues and Vices of Vocal Translation. Brill, Netherlands. 2005. [7] A. Chan, A, Noble. Sounds in Translation: Intersections of Music, Technology and Society. ANU E Press. 2009.

[8] H. J. Minors. Music, Text and Translation. London: Bloomsbury Academic. 2013.

[9] R. Apter, M. Herman. Translating for Singing: the Theory, Art and Craft of Translating Lyrics. Bloomsbury Academic. 2016.

[10] P. Low. Translating Song: Lyrics and Texts. London: Routledge. 2017.

[11] 黄忠廉.变译理论[M].北京: 中国对外翻译出版公司, 2001:78, 96. 
[12] 黄忠廉.变译理论: 一种全新的翻译理论[J].国外外语教学, 2002（1）：19-22. [13] 杨晓静.歌曲翻译三符变化说——以俄语歌曲汉译为例[D].黑龙江大学, 2012. 